\title{
African Yearbook of International Law / Annuaire Africain de Droit International,
}

Vol. 3 (1995)

Published under the auspices of the African Association of International Law, edited by Abdulqawi A. Yusuf

Kluwer Law International, The Hague, London, Boston, 463 pp., £130.00

Der 3. Band des Jahrbuchs (zu den Bänden 1 und 2 siehe VRÜ 29, S. 95 ff. bzw. 30, S. 254 f.) behandelt als Spezialthema Menschenrechte und Entwicklung. Nach kürzeren Reflektionen von Abi-Saab zum Zusammenhang beider Begriffe und von Boutros-Ghali über die Vereinten Nationen und das Ziel der Demokratisierung ("No pre-determined models can be superimposed") findet sich eine Abhandlung von Kwakwa (Ghana, UNHCR), welche Zusammenhänge zwischen der Flüchtlingsproblematik ("Population displacement") und der Realisierbarkeit entwicklungsorientierter Politik untersucht, faktenund materialreich. Maluwa behandelt mit menschenrechtlichem Schwerpunkt das Verhältnis von Völkerrecht und malawischem Recht. Maina Peter behandelt kritisch bis sarkastisch die Frage nach der Menschenrechtsdurchsetzung in Tansania. Adede gibt eine Skizze vertraglicher Entwicklungen im Menschenrechtsbereich. Meliboute beschäftigt sich anhand afrikanischer Fälle mit humanitärem Völkerrecht.

Neben weiteren Aufsätzen dokumentiert das Jahrbuch das UNO-Verhalten insbesondere 1994/1995 zu verschiedenen inneren Konflikten in afrikanischen Staaten sowie die Resolutionspraxis der OAU in den beiden Jahren. Im Rezensionsteil fällt die freundliche Präsentation von John Dugards Völkerrechtslehrbuch "in südafrikanischer Perspektive" durch Muna Ndulo auf.- Das Jahrbuch scheint sich etabliert zu haben. Thematisch wie personell dominiert wie in den vorigen Bänden die UNO. Das ist bezeichnend.

Philip Kunig

Robert Alexy / Ralf Dreier (Hrsg.)

ARSP - Archiv für Rechts- und Sozialphilosophie "Rechtssystem und praktische Vernunft"

$\mathrm{XV}$. Weltkongreß der internationalen Vereinigung für Rechts- und Sozialphilosophie (IVR), Göttingen, 18. bis 24. August 1991, Band 1, Vol. 1, Beiheft Nr. 51

Franz Steiner Verlag, Stuttgart, 1993, 313 S., DM 120,--

Philosophie und Recht - ein spannendes Feld, und wenn sich die Rechtswissenschaft zur Philosophie hinwendet, läßt dies vieles an Erkenntnisgewinn erhoffen. Denn schließlich ist es doch notwendig, sich hin und wieder zu besinnen, nachzudenken, einiges zu bedenken und vieles vorzudenken - schlicht das zu tun, was die Philosophie als ursprünglichstes tut - 
überhaupt denken. ${ }^{1}$ Der vorliegende Band nun beinhaltet auf 310 Seiten 28 kurze Vorträge in drei Sprachen (deutsch, englisch, spanisch) vom XV. Weltkongreß 1991der IVR. ${ }^{2}$ Der Band hat drei Kapitel: Im ersten Kapitel sind zwei Plenarvorträge von Robert Alexy und Ota Weinberger abgedruckt, in denen es um die "praktische Vernunft" geht, ein öffentlicher Vortrag (Kapitel 2) handelt von der Universalität der Menschenrechte (Martin Kriele) und die übrigen Texte (Kapitel 3) von Einzelautoren sind die Arbeitsgruppenreferate, die sich dem Thema "praktische Vernunft" von vielen Seiten her zu nähern suchen, sei es aus feministischer Sicht, sei es aus der Perspektive der Postmoderne oder des Utilitarismus.

Die Auswahl dieses eigentlich nicht mehr ganz druckfrischen Bandes für eine Rezension ist zwar eher einem Zufall geschuldet, ist aber angesichts eines ganz bestimmten Aspektes, der im folgenden noch ausgeführt werden wird, höchst aktuell. Schon ein flüchtiger Blick des wissenschaftlich Interessierten auf die Quellenlage der einzelnen Vorträge erweckt aus zwei Gründen Aufmerksamkeit: Erstens ist dieser Band eine Zusammenstellung eines bestimmten rechtstheoretischen Ansatzes, dessen Konturen Robert Alexy, Mitherausgeber des Archivs, gleich zu Beginn im ersten Kapitel darlegt. Die weiteren Autoren verarbeiten eigentlich nur Alexys Thesen. Aus diesem Grunde erscheint es angemessen, an dieser Stelle vornehmlich den Aufsatz von Alexy (S. 9 ff.) pars pro toto zu besprechen. Zweitens fällt schon beim ersten Durchblättern auf, daß sich, obwohl das Kongreßthema sowohl Recht als auch Philosophie umfassen soll, philosophisches Gedankengut nur in der oben erwähnten Perspektive findet. Eine kritische Auseinandersetzung anhand von Texten philosophischer Autoren wie etwa Plato von Athen, Aristoteles, oder eventuell Cicero, Augustinus oder gar Thomas von Aquin, Hobbes, Wittgenstein, die sehr wohl und intensiv über das Recht nachgedacht haben, findet eher nicht statt. ${ }^{3}$ Und dies stimmt aus philosophischer Perspektive sehr nachdenklich, war man doch zunächst gespannt, welche notwendigen und interessanten Gedanken sich aus der Interdisziplinarität beider Wissenschaften ergeben würden.

1

"In das, was denken heißt, gelangen wir, wenn wir selber denken. Damit ein solcher Versuch glückt, müssen wir bereit sein, denken zu lemen. Sobald wir uns auf dieses Lemen einlassen, haben wir auch schon zugestanden, daß wir das denken noch nicht vermögen." (Heidegger, Martin, Was heißt denken?, Stuttgart 1994, 3). Erst aus dieser Bescheidenheit des Bewußtseins ist der Beginn der Philosophie erst möglich. Sinn philosophischen Denkens ist Erkenntnisgewinn.

Dieser Kongreß steht in der Tradition des im Jahre 1907 von Joseph Kohler und Fritz Berolzheimer begründeten "Archivs für Rechts- und Wirtschaftsphilosophie". 1911 wurde in diesem Zusammenhang die "Internationale Vereinigung für Rechts- und Sozialphilosophie" gegründet, in der heute über vierzig nationale Vereinigungen zusammengeschlossen sind. Die damit verbundene Intermationalisierung deutet auf einen zunehmenden Anteil außerrechtlicher Methoden in der und Einflüssen auf die Rechtsphilosophie hin. Vgl. Ritter, Joachim, Historisches Wörterbuch der Philosophie, Stuttgart 1967 ff., Stichwort: Rechtsphilosophie.

3

... um nur ein paar zu erwähnen, mit denen man eine Auseinandersetzung hätte erwarten können: Xenophon, Memorabilien (Hippias); Platon, Dialoge Trasymachos und Theaitetos; Aristoteles, Nikomachische Ethik und Politik; Seneca, Epistulae ad Lucilium; Gaius, Institutiones; Codex Juris Canonici (Decretium Gratiani); Augustinus, De civitate dei; Thomas von Aquin, Summa Theologica; Immanuel Kant, Metaphysik der Sitten und Einleitung in die Rechtslehre; Georg Wilhelm Friedrich Hegel, System der Philosophie und Grundlinien der Philosophie des Rechts. 
Auf der Suche nach den Ursachen für dieses Phänomen fällt folgendes auf: Die Wissenschaft Philosophie ${ }^{4}$ steht zur Rechtswissenschaft in einem schwierigen Verhältnis. Die Schwierigkeit dieses Verhältnisses liegt in einem ganz bestimmten Punkt und der vorliegende Band ist ein klassisches Beispiel dafür: Wer in einem geschlossenen System denkt, muß nicht nach den Gründen der Axiome des Systems fragen - es genügt, wenn er diese hinnimmt und sie anwendet. Anders dagegen verhält es sich in der Philosophie: Sie weist über Systeme hinaus - dieses geistige Ethos erst gebiert die Möglichkeit weiterer Erkenntnis. Die vorliegende Diskussion um Thesen, insoweit sie die Philosophie berühren, nähern sich diesem Ethos der Philosophie nicht. Denn das Rechtssystem wird nicht etwa auf der Folie der Philosophie untersucht, sondern es wird die Philosophie als Steinbruch für eigene Theoreme genutzt. Aus wissenschaftstheoretischer Sicht begibt man sich damit um die Möglichkeiten der "zur Hilfe genommenen" Wissenschaft und damit um wirkliche Interdisziplinarität. So wurden trotz der Vielfalt von bedenkenswerten Ansätzen der Philosophiegeschichte hinsichtlich des Rechts in diesem Band lediglich zwei - und dies auch nur schemenhaft - Ansätze zur Klärung rechtsphilosophischer Untersuchungen herangezogen: Praktische Vernunft und Diskurstheorie.

In seinem Vortrag nun hebt Alexy seine Ausführungen zum Thema mit einer "diskurstheoretischen Konzeption der praktischen Vernunft" an. Sein erster Ansatz ist es, zu zeigen, daß es eine praktische Vernunft nicht gibt. Eine Begründung oder Erklärung wird dazu nicht weiter gegeben, vielmehr wird die als "kantische Theorie" bezeichnete praktische Vernunft durch die Diskurstheorie für überwunden erklärt, ohne daß etwa auch auf die Diskurstheorie näher eingegangen würde. Zur Vereinfachung werden praktische Vernunft und praktische Rationalität gleich gesetzt. ${ }^{5}$ Nachdem es also keine praktische Vernunft mehr gibt, wird die Diskurstheorie als Prozedur eines kommunikativen Interessenausgleichs ${ }^{6}$ definiert, wobei allgemein verbindliche Rechtsnormen Ergebnis solcher Prozeduren sein sollen. Und so ist scheinbar die Quelle des Rechts - auch in seiner möglicherweise ethischen Qualität durch eine schlichte Prozedur des Interessenausgleichs herleitbar. Dadurch scheint es möglich, daß "die Diskurstheorie zeigen kann, wie praktische Vernunft wirklich werden kann,

Griechisch (gr.), Liebe zur Weisheit. Die wichtigsten Disziplinen der Philosophie sind: Logik, Erkenntnistheorie, Ethik, Metaphysik, Ästhetik; darüber hinaus gibt es die Einzelphilosophien: 5 Geschichtsphilosophie, Philosophie der Kunst, Rechtsphilosophie, Sozialphilosophie u.a.

... wobei Rationalität schon bei Apuleius schlichte vox docta ist, die sich der Vernunft bediente. S. Apuleius von Madaura, De dogmate Platonis, 2,15, hrsg. von P. Thomas, 1970, 118; vgl. auch die philosophische Unterscheidung zwischen bloßer Meinung und wirklicher Erkenntnis.

6

Interesse - aus lat. inter esse - dazwischen sein; Interesse wird hier lediglich materialistisch im Sinne einer größtmöglich ausgeglichenen Verteilung von Gütern und Rechten usw. - also wie etwa das, was Heidegger das "Interessante" nennt, aufgefaßt: "Inter-esse [aber] heißt: unter und zwischen den Sachen sein, mitten in einer Sache stehen und bei ihr bleiben. Allein für das heutige Interesse gilt nur das Interessante. Das ist solches, was erlaubt, im nächsten Augenblick schon gleichgültig zu sein und durch anderes abgelöst zu werden, was einen dann ebensowenig angeht wie das vorherige." S. Heidegger, a.a.O., 5. 
ohne unterschiedlichen Lebensformen und Konzeptionen des guten Lebens den Respekt zu versagen" (S. 29). [Der banale Umkehrschluß könnte dann - frei nach Alexy - sein, daß die praktische Vernunft gutes Leben verhindert, und die Prozedur eines Interessenausgleichs gutes Leben erst ermöglicht. Philosophisch betrachtet ist dies eine eher recht schlichte Vorstellung zu einer Konzeption des "guten Lebens". ${ }^{7}$ ]

Aber zurück zum Recht als System im Verhältnis zur Philosophie als universaler Wissenschaft. Der Systembegriff ist recht schwierig, eines aber steht fest: System ist stets eine Zusammensetzung verschiedener Entitäten. ${ }^{8}$ Der Begriff "System" bezeichnet eine "strukturierte Einheit, ein geordnetes Ganzes, dessen Teile nach bestimmten Regeln, Gesetzen oder Prinzipien ineinandergreifen." ${ }^{9}$ System meint im weiteren Sinne eine "Architektonik [... also...] die systematische Einheit [desjenigen], was gemeine Erkenntnis allererst zur Wissenschaft, d.i. aus einem bloßen Aggregat derselben ein System macht. ... System [ist] die Einheit der mannigfaltigen Erkenntnisse unter eine Idee." ${ }^{10}$ System verweist insofern auf eine übergeordnete "Einheit" mit einer gewissen Mannigfaltigkeit. Trotzdem ist der Begriff des Systems gerade nicht ein Begriff der Transzendentalphilosophie wie etwa der Begriff der praktischen Vernunft, denn er verweist lediglich auf eine einheitliche, konkrete Ordnung aber keineswegs etwa auf das Allgemeine (Universale) an sich.

Gegen den transzendentalen Begriff der praktischen Vernunft versucht Alexy nun, für das Rechtssystem den Begriff der "Richtigkeit" zu setzen, und stellt das Postulat auf, daß "die Idee praktischer Richtigkeit die Idee der Verallgemeinerbarkeit einschließt" (S. 28). Obwohl Alexy dieses Postulat nicht weiter begründet, könnte man nun vermuten, daß er den Begriff der Richtigkeit nachdrücklich in der Qualität eines transzendentalphilosophischen Begriffes verstanden wissen möchte, wenn er diesen Begriff auf der Folie der Trandszendentalphilosophie diskutiert. Um so weniger ist es dann ersichtlich, wieso denn im Zusammenhang einer rechtsphilosophischen disputatio, die ein solcher Kongreß sein

Hierzu auch Aristoteles, Metaphysik 1021 b 21 ff.; Physik a 13 - 17; Nikomachische Ethik 1098 b2. Wenigstens ein Hinweis auf Epikur, Seneca, Dilthey oder Bergson sollte hier nicht fehlen.

Gr. für "Zusammensetzung". Das entspricht in etwa dem lateinischen Begriff compositum. In der formalen Logik unterscheidet man abstrakte, informationslogische, kontradiktorische, äquipollente, kovariante Systeme, die mathematische Logik versteht unter einem algebraischen System die Struktur formalisierter Theorien und Kalküle. Vgl. Kondakov, N.I., Logik, Berlin, 1978, System. - entitas - "Seiendheit": In ontologischer "Relation" verhält sich das Seiende gegenüber dem "Sein" wie das erfahrene Objekt gegenüber dem Wesen, quasi seiner selbst. Erst in der metaphysischen Reflexion wird - und dies ist die Aufgabe der prima philosophia - das "Seiende als Seiendes in seinem Inneren Sinn und Aufbau" erschlossen. Durch diese Reflexion "gelangen wir erst zu den jedem Gegenstand logisch vorgeordneten inneren Prinzipien der Gegenständlichkeit, zu den inneren Gründen der Möglichkeit der Phänomene und ihrer Erfahrung, zu den substantiellen Seinsprinzipien, die Einheit und Ordnung, Sinnerfülltheit und werthafte Zielbestimmtheit der Phänomene und ihrer Erkenntnis vorherbestimmen." Vgl. Nink, Caspar, Ontologie, Freiburg 1950, 1.

9 Hïgli/Lübcke, a.a.O. (System). 
will, andere transzendentalphilosophische Denkansätze wie zum Beispiel die kritische Philosophie Kants verworfen werden müssen, nur weil man gleichzeitig ein eigenes Theorem der "Richtigkeit der Durchsetzung von individuellen Interessen" als die "wahre" Theorie der Begründung von Rechtsprinzipien einführen möchte. ${ }^{11}$

Es läßt sich zusammenfassen, daß im vorliegenden Band eigentlich bei fast allen der Autoren praktische Vernunft als transzendentales a priori ebenso nicht beachtet wurde wie etwa die in der Tradition der Transzendentalphilosophie stehende Diskurstheorie als das wissenschaftliche Bemühen um allgemeingültige Sätze und vielleicht Quelle derselben. ${ }^{12}$ Wenn der kantische Vernunftbegriff radikal auf den Begriff der Rationalität, der ja - ganz im Gegensatz zu Kants Vernunftbegriff - lediglich ein Begriff der klassischen Ökonomie ist, reduziert wird, und Diskurstheorie lediglich als Interessenausgleichsprozedur, also als die Ausprägung und das Einhalten bestimmter Regeln für eine Diskussion, interpretiert wird, macht das Postulat einer Idee der Richtigkeit nur in einer ideologisch fixierten Betrachtung des Rechts überhaupt einen Sinn. Es ist eigentlich unnötig, wenn nicht gar unlogisch, transzendalphilosophische Elemente auf diese Weise zu verarbeiten, es sei denn, man glaubt seine Thesen gegenüber der zuvor verworfenen Transzendentalphilosophie rechtfertigen zu müssen, was ihre Bedeutung für das eigene Theorem - ganz abgesehen von ihrer wahren Bedeutung für die abendländischen Geistesgeschichte - erheblich steigern würde. Es fragt sich darüber hinaus, ob angesichts des Kongreßthemas die Reduzierung der Transzendentalphilosophie auf ein Theorem der praktischen Vernunft ${ }^{13}$ im Sinne einer Interessenausgleichsprozedur überhaupt fachlich zulässig ist und es wird nicht klar, welchen Erkenntnisgewinn das für ein konkretes Rechtssystem mit sich bringen könnte. Man setzt

11

... und dies angesichts der Tatsache, daß spätestens seit Anselm von Canterbury die systematische Verknüpfung von Richtigkeit und Wahrheit aufgegeben worden ist. Vgl. Anselm von Canterbury, de veritate 1, 191, Zitationsziffern nach der Gesamtausgabe von Gabriel Gerberon, Paris 1675. Vgl. zu dieser Problematik auch von Cherbury, Herbert, De veritate, prout distinguitur a relatione, a verilissimi, a possibili, et a falso, Erstausgabe, Paris 1624. Immerhin gesteht Alexy sowohl Immanuel Kant wie auch Vertretern der Diskurstheorie wie etwa Habermas oder Apel jovial zu, daß ihnen gewisse Leistungen nicht abgesprochen werden können. Denn die kantische "praktische Rationalität", so die Erläuterung, habe die Menschenrechte hervorgebracht und die Diskurstheorie die "Prozedur". Es sollte aber an dieser Stelle nicht unerwähnt bleiben, daß Niklas Luhmann mit seiner Systemtheorie, die ja hier insgeheim rezipiert wird, methodisch keineswegs so salopp mit einer der größten Traditionen der abendländischen Philosophie umgeht. Vgl. Luhmann, Niklas, Rechtssoziologie, 1972.

Vgl. dazu näheres bei: Apel, Karl-Otto, Transformation der Philosophie, 1973.

13 Schließlich ist noch eine begriffliche Ungenauigkeit, die den gesamten Band durchzieht, anzumerken: Hier wird stets als "praktische Vernunft" bezeichnet, was von den Vertretern der Diskurstheorie (Adorno, Habermas, Horkheimer), auf die ja hier eingegangen werden soll, mit dem Begriff "instrumentelle Vernunft" bezeichnet wird. Instrumentelle Vernunft zielt auf die "Beherrschung der Wirklichkeit ab. (...) Diese sollten von einem auf Konsens ausgerichteten und auf gegenseitiger Anerkennung beruhenden Diskurs ausgehen." Vgl. Hiigli, a.a.O., Lemma: Vernunft sowie Habermas, Jïrgen, Erkenntnis und Interesse, Frankfurt 1968; Adorno,Theodor / Horkheimer, Max, Dialektik der Aufklärung, New York 1944, Frankfurt 1947. 
sich, nimmt man die Zulässigkeit trotzdem an, einem Ideologieverdacht in Richtung einer rein dogmatischen Rechtstheorie aus, woraus zu schließen ist, daß hier die Philosophie im Sinne solcher Rechtsideologie instrumentalisiert werden soll. Doch dieser Umgang mit der Philosophie tut ihr ganz und gar "un"-"recht".

Guntram Platter

\section{Peter Saladin}

Wozu noch Staaten?

$\mathrm{Zu}$ den Funktionen eines modernen demokratischen Rechtsstaats in einer zunehmend überstaatlichen Welt

Stämpfli, Bern, C.H. Beck, München, Manz'sche Verlags- und Universitätsbuchhandlung, Wien, 1995, 249 S., DM 98,--

Dieses Buch ist Peter Saladins, des bedeutenden Schweizer Staatsrechtlers, Vermächtnis geworden. Es ist die letzte größere Arbeit des im vergangenen Jahr verstorbenen Wissenschaftlers. Die Rede vom "Staatsversagen" sowie die "Internationalisierung und Supranationalisierung des öf fentlichen (und in gewisser Hinsicht auch des privaten!) Lebens" (S. 14) sind seine Anlässe, staatstheoretisch die Beobachtung, daß die Vorstellungen über Staatsgebiet, Staatsvolk, Staatsgewalt ihre "Selbstverständlichkeit" eingebüßt hätten (ebenda). Dem geht die Schrift zu Beginn pointiert und mit Beispielen nach, oft aus der Schweizer Politik. Sie untersucht sodann, was den Staat heute "eigentlich" kennzeichne, was seine spezifische Rolle im Zusammenwirken mit anderen Akteuren sei, welche Konsequenzen für Rechtsstaatlichkeit, Demokratie und Ordnungsbewahrung folgen. Vor allem für die Schweiz, Deutschland und Österreich werden Staatsaufgaben analysiert, je in Zuordnung zu "Bund", Gliedern, Kommunen und unter Berücksichtigung der EU und auch anderer Organisationen. Dabei macht Saladin einen Aufgabenverlust des Staates aus, was ihm Anlaß ist, nach dem Kern von Aufgaben zu fragen, die staatlicher Wahrnehmung vorbehalten sein sollten. Das führt zu methodisch angeleiteten und anhand differenzierter Kriterien vorgenommenen Aufgabenzuordnungen, wobei der Autor sämtliche Politikagenden berührt und eindrucksvoll in Rechnung stellt, daß der Staat von ihm wahrgenommene Aufgaben zunehmend in anderen Formen als denen vor dem Hintergrund potentiellen Zwangs gesetzter Befehle erfüllt, nämlich inspirierend, anreizend, kooperierend. Die dichte Untersuchung wird hingeführt zu dem Bild des Staates als einer "Brücke", eines "Mittlers" zwischen den "überstaatlichen" und den "unterstaatlichen" Ebenen. Mit ihm eigener unprätentiöser Nonchalance bringt der Autor freilich selbst zum Ausdruck, diese Begriffe und von ihnen erweckte Assoziationen möchten "mißverständlich" oder auch "schief" sein. Die Brücke verbinde nicht nur, sondern sei im Sinne des "Ponte Vecchio" zu denken, also mit einer "Siedlung". Es sind dies nicht nur kultivierte Sprachspiele. Saladin nutzt solche 